\title{
Long noncoding RNA IncHERG promotes cell proliferation, migration and invasion in glioblastoma
}

\author{
Jian Shi ${ }^{1}$, Yong-Jie Wang ${ }^{1}$, Chong-Ran Sun ${ }^{1}$, Bin Qin ${ }^{1}$, Yang Zhang ${ }^{1}$ and Gao Chen ${ }^{1}$ \\ ${ }^{1}$ Department of Neurosurgery, The Second Affiliated Hospital of Zhejiang University School of Medicine, Hangzhou 310000, \\ China \\ Correspondence to: Jian Shi, email: shijian201511@126.com \\ Gao Chen, email: d-chengao@zju.edu.cn \\ Keywords: InCHERG; proliferation; migration; miR-940; glioblastoma \\ Received: September 29, $2017 \quad$ Accepted: October 27, $2017 \quad$ Published: November 14, 2017 \\ Copyright: Shi et al. This is an open-access article distributed under the terms of the Creative Commons Attribution License 3.0 (CC \\ BY 3.0), which permits unrestricted use, distribution, and reproduction in any medium, provided the original author and source are \\ credited.
}

\section{ABSTRACT}

Long noncoding RNAs have recently been proven to regulate tumorgenesis in many cancers. However, their biological functions in glioblastoma remain largely unknown. Here we found an uncharacteristic IncRNA IncHERG that is highly expressed in human glioblastoma (GBM). We found that IncHERG knockdown inhibited cell proliferation, migration and invasion in glioblastoma in vitro and in vivo. Moreover, the higher expression of IncHERG in patients with glioblastoma indicated lower survival rate and poorer prognosis. Mechanistically, we found that IncHERG can serve as a sponge for miR-940 which is a tumor suppressor in cervical cancer and whose function has not been defined in glioblastoma. We showed that miR-940 was down-regulated in glioblastoma tissues compared to peritumor tissues. LncHERG knockdown impaired cell proliferation, migration and invasion while inhibition of miR-940 in the meantime reversed this trend. In conclusion, our study highlights the essential role of IncHERG in glioblastoma by acting as a competing endogenous RNA of miR-940, which may serve as a new prognostic biomarker in glioblastoma.

\section{INTRODUCTION}

Glioblastoma (GBM), the most common and aggressive brain tumor around the world, leads to a large amount of deaths each year $[1,2]$. The incidence of glioblastoma is increasing year by year. Surgical resection, radiotherapy and chemotherapy were the main methods for treatment of GBM patients [3-6]. However, the 5-year overall survival rate of patients with GBM is less than $3 \%[7,8]$. Due to the infiltrative growth of glioblastoma, the rate of tumor recurrence is very high [5]. There is no effective method for fully treatment of glioblastoma so far. Therefore, in order to develop new and effective therapeutic management of GBM, it is an urgent and necessary to define the molecular mechanism that regulates the genesis of glioblastoma.

Long noncoding RNAs (lncRNA) are a class of transcripts whose length is longer than 200 nucleotides and have no protein coding potential [9]. Accumulating evidences show that noncoding RNAs (ncRNA) exert essential roles in all kinds of biological processes including development, immune and especially in tumorgenesis [10-13]. In cancers, lncRNAs have been demonstrated to regulate cell proliferation, migration, invasion and apoptosis by several mechanisms [14]. A lot of lncRNAs are up-regulated or down-regulated in cancers and involved in tumor development and progression [15]. For example, lncRNAAFAP1-AS1 increases tumor growth and metastasis in HCC [16]. LncRNA DUXAP8 promotes cell proliferation and migration in gastric cancer [17]. LncRNA UCA1 promotes gallbladder cancer progression via inhibiting p21 and E-cadherin expression [18]. LncRNA CCEPR is upregulated and associated with poor prognosis in urothelial bladder carcinoma [19]. In glioblastoma, lncRNA HOXAAS3 increases tumor progression and lncRNA TALNEC2 promotes growth of glioblastoma stem cells [6, 20]. Nevertheless, there are still many uncharacteristic lncRNAs whose functions remain to be illustrated in glioblastoma. Hence, to understand the molecular biology of glioblastoma, it is important to identify more tumor-related lncRNAs. 
In our study, we found an uncharacteristic lncRNA lncHERG (Highly expressed long noncoding RNA in glioblastoma) whose expression was higher in glioblastoma than non-tumor tissues. We showed that lncHERG depletion impaired proliferation, migration and invasion of glioblastoma in vitro and in vivo. What's more, the higher expression of IncHERG in patients with glioblastoma indicated lower survival rate and poorer prognosis. In mechanism, we found that IncHERG sponged miR-940 in glioblastoma. Moreover, miR-940 was down-regulated in glioblastoma tissues compared to peritumor tissues. LncHERG knockdown impaired cell proliferation, migration and invasion while inhibition of miR-940 in the meantime reversed it. In summary, our study highlights the essential role of $\operatorname{lncHERG}$ in glioblastoma by acting as a competing endogenous RNA of miR-940, which may serve as a new prognostic biomarker in glioblastoma.

\section{RESULTS}

\section{LncHERG is highly expressed in glioblastoma}

To explore the biological functions of lncRNAs in glioblastoma, we analyzed an online dataset (GSE90598). We found out an uncharacteristic lncRNA (official symbol: LOC644794) lncHERG that was up-regulated in GBM tissues compared to non-tumor tissues (Figure 1A). lncHERG was significantly up-regulated in glioblastoma (Figure 1B) according to this dataset (GSE90598). To confirm this result, we collected glioblastoma samples and extracted total RNAs. Then we checked the relative expression levels of lncHERG in 20 pairs of glioblastoma samples. We found that lncHERG was also remarkably upregulated in most glioblastoma samples (Figure 1C). Then we checked the expression of IncHERG in normal glial cell line (HA) and GBM cell lines (U87, U251 and
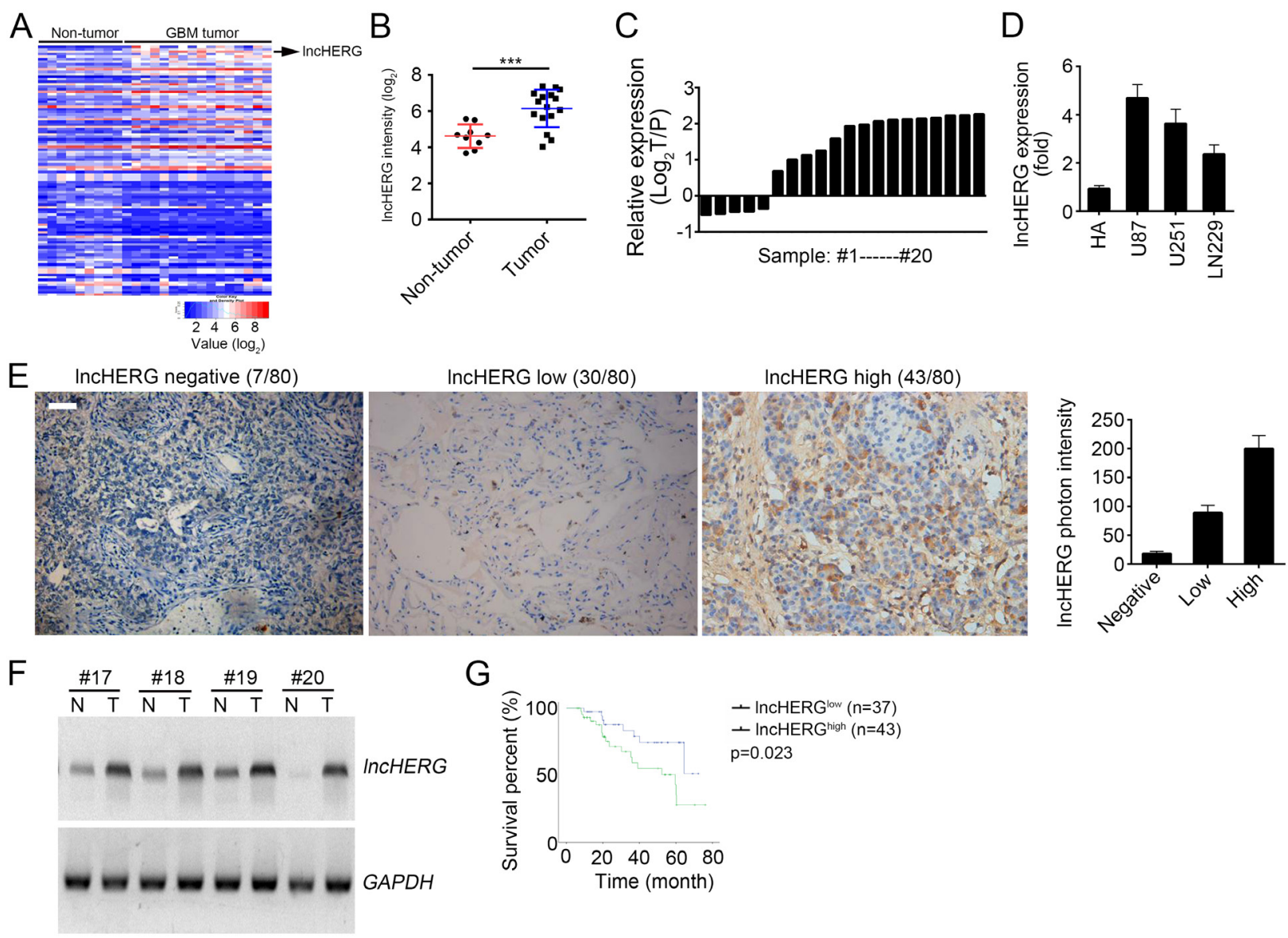

Figure 1: LncHERG is highly expressed in glioblastoma. (A) LncHERG was up-regulated in glioblastoma (GBM) tissues according to a microarray dataset (GSE90598). (B) Analysis of lncHERG expression in non-tumor and GBM tissues according to dataset in GSE90598. (C) The expression levels of lncHERG in GBM tissues and paired peritumor tissues were measured. (D) The expression of lncHERG in normal glial cell line (HA) and GBM cell lines (U87, U251 and LN229) was analyzed. (E) The expression levels of lncHERG in GBM tissues were checked by in situ hybridization (ISH). lncHERG was highly expressed in GBM tissues. (F) Total RNAs were obtained from GBM tissues and paired peritumor tissues. And lncHERG expression was measured by Northern blot. N, non-tumor; T, Tumor. (G) 80 GBM samples were collected and divided into two groups based on lncHERG expression (cut-off: mean value). Then the overall survival rate was analyzed by Kaplan-Meier analysis. All data are representative of three independent experiments and expressed as mean \pm SD. ${ }^{* * *} p<0.001$. 
LN229). IncHERG was highly expressed in GBM cell lines (Figure 1D). To further validate it, we performed in situ hybridization (ISH) assays with specific biotinlabeled probes in 80 glioblastoma samples. We found that most glioblastoma samples showed very high expression of lncHERG (Figure 1E). What's more, we conducted Northern blot assays. As shown, lncHERG was also highly expressed in glioblastoma samples (Figure 1F). Then we divided these samples into two groups according to lncHERG expression levels (mean value was the cutoff). We performed Kaplan-Meier analysis and found that patients with higher lncHERG expression showed lower overall survival rate (Figure $1 \mathrm{G}$ ). In summary, lncHERG was highly expressed in glioblastoma samples and correlated with tumor prognosis.

\section{LncHERG promotes cell proliferation and inhibits cell apoptosis}

To define the physiological function of lncHERG in glioblastoma, we knocked down lncHERG in U87 and U251 cells (Figure 2A). Then we performed MTT assays using lncHERG-depleted stable cell lines. We found that lncHERG knockdown significantly inhibited
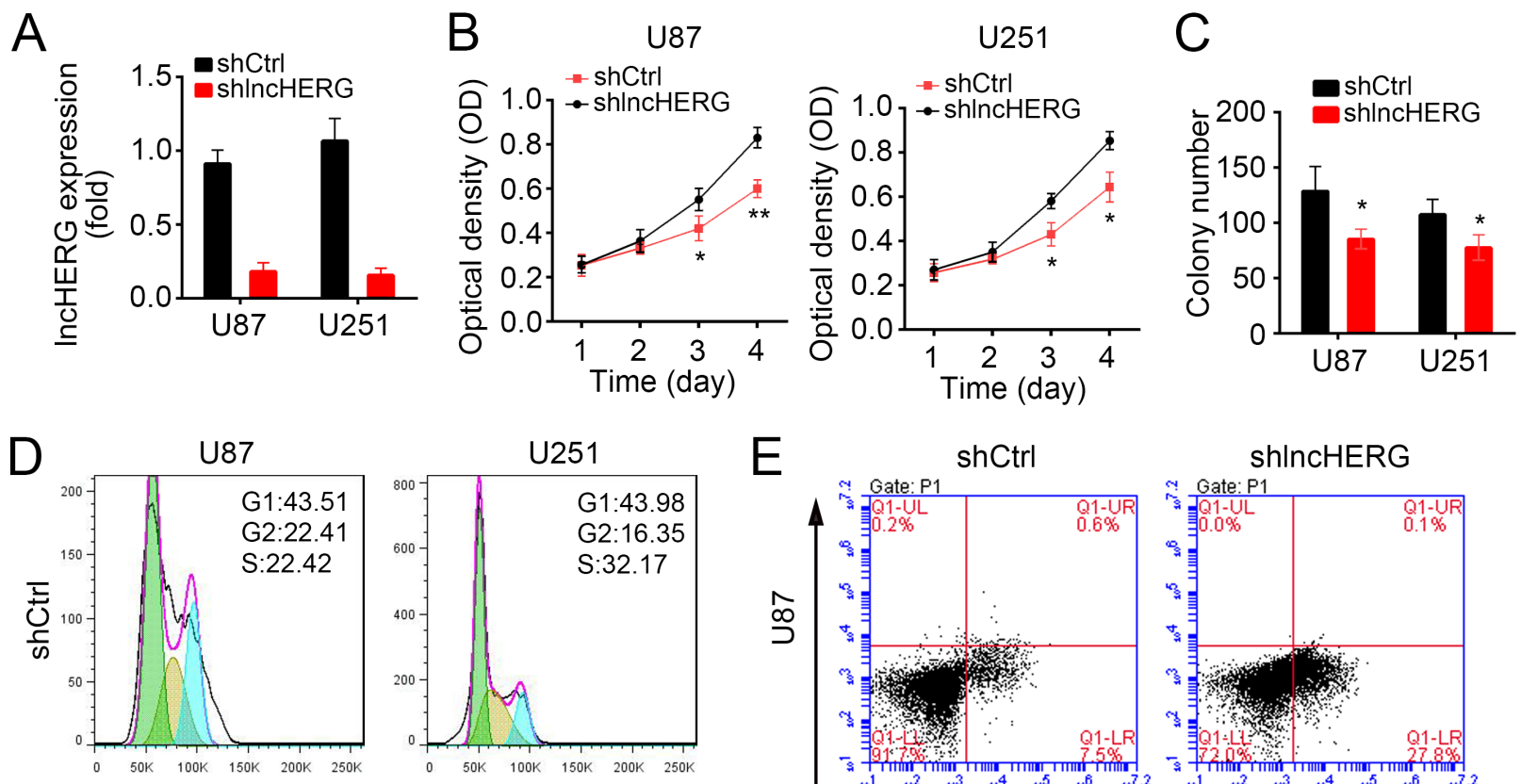

E
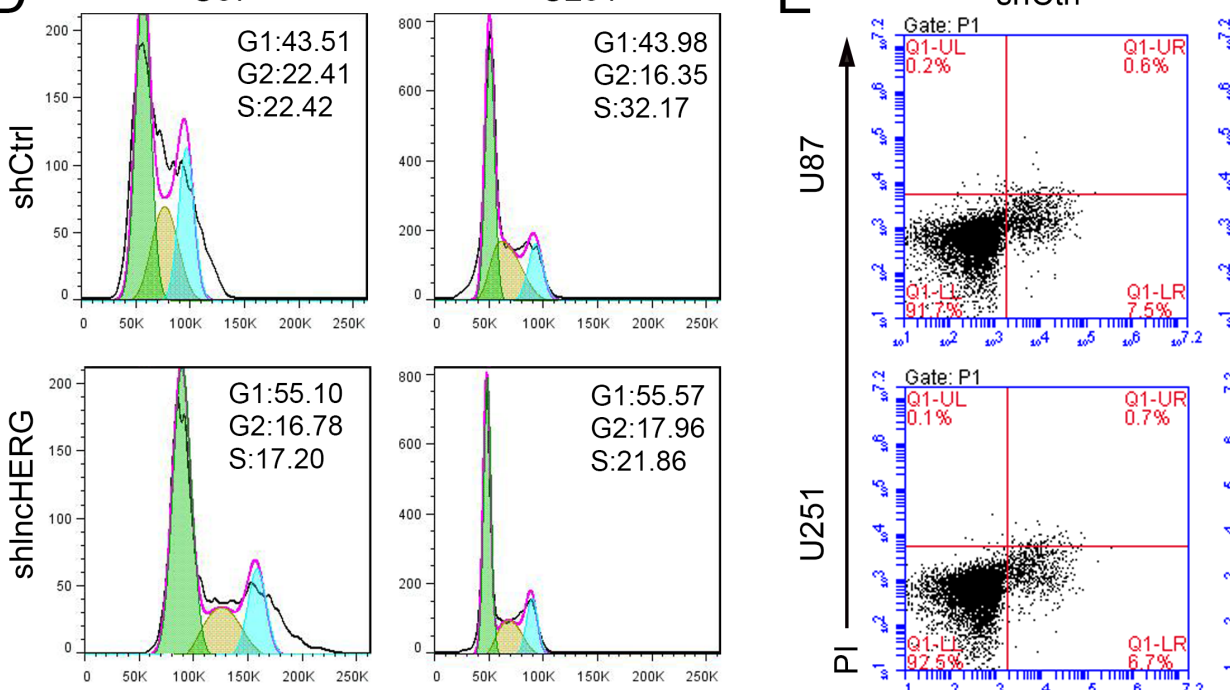

ShCtrl
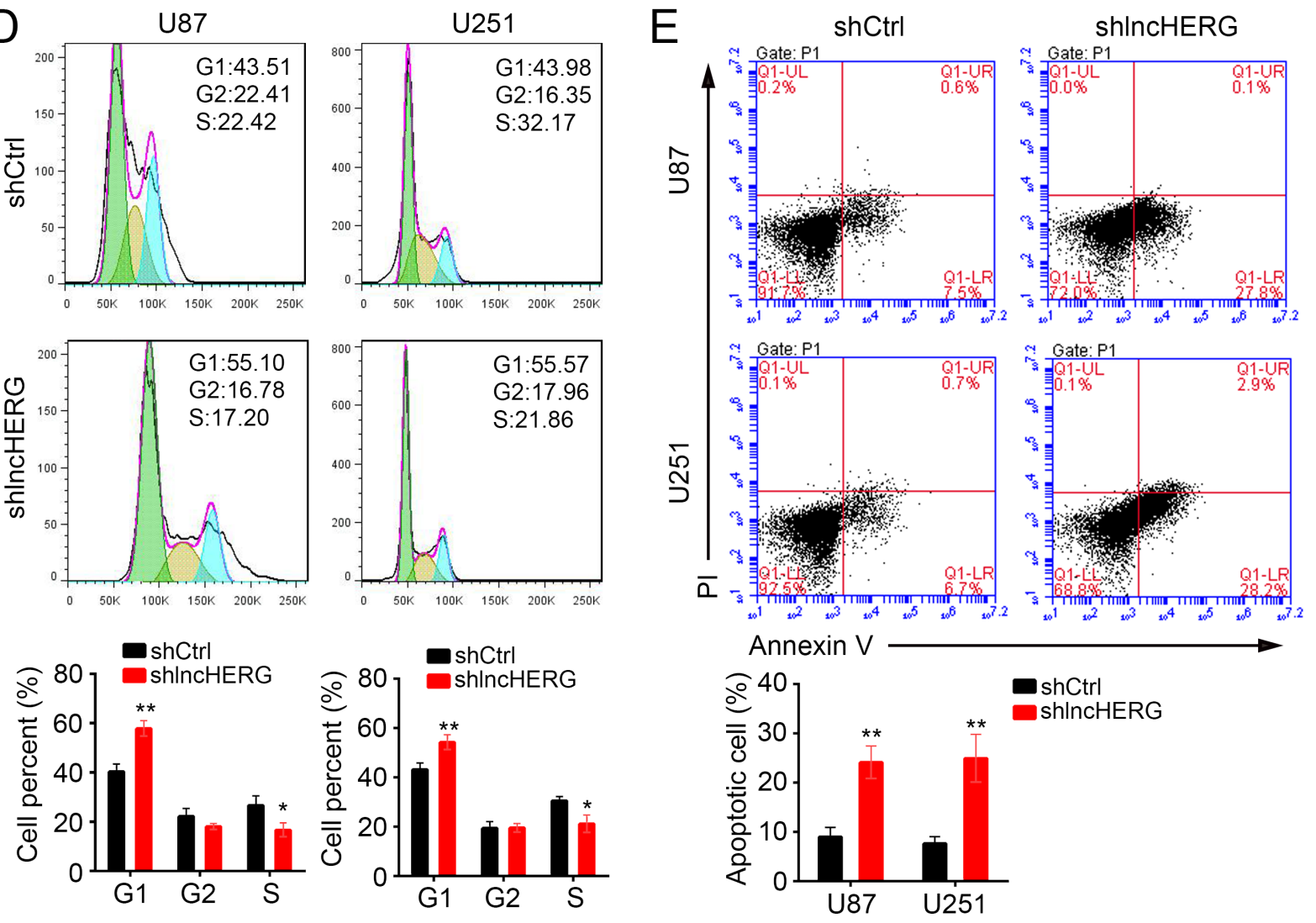

Figure 2: LncHERG promotes cell proliferation and inhibits cell apoptosis. (A) LncHERG was knocked down by transfection with specific shRNA. The mRNA levels were analyzed by qPCR in lncHERG-depleted cells. (B) MTT assays were conducted with shCtrl or shlncHERG U87 and U251 cells. LncHERG knockdown remarkably inhibited cell proliferation. (C) LncHERG knockdown impaired the abilities of colony formation of U87 and U251 cells. (D) less LncHERG-silenced cells entered into S phase. (E) LncHERG depletion dramatically increased the cell apoptosis in U87 and U251 cells. All data are representative of three independent experiments and expressed as mean $\pm \mathrm{SD}$. ${ }^{*} p<0.05$ and ${ }^{* *} p<0.01$. 
cell proliferation (Figure 2B). What's more, lncHERGsilenced cells formed less clones than shCtrl cells (Figure 2C). Consistently, lncHERG knockdown remarkably impaired cell division. Less shlncHERG cells entered into $\mathrm{S}$ phase (Figure 2D). Then we evaluated the cell apoptosis by Annexin V/PI staining. We found that lncHERG silence promoted cell death in U87 and U251 cells (Figure 2E). Collectively, lncHERG enhanced cell proliferation and decreased cell apoptosis in glioblastoma.

\section{LncHERG enhances cell migration and invasion}

Tumor metastasis is the main cause for cancer recurrence and malignance. We wondered whether lncHERG regulated tumor metastasis in glioblastoma. We performed cell migration and invasion assays. After lncHERG knockdown, the abilities of migration and invasion of U87 and U251 cells were dramatically impaired (Figure 3A and 3B). In consistence, the expression levels of metastasis-related proteins (TWIST1, SNAIL and MMP9) were remarkably down-regulated after lncHERG knockdown in U87 and U251 cells (Figure 3C). In summary, lncHERG promoted tumor metastasis in glioblastoma.

\section{LncHERG depletion impairs tumor propagation in vivo}

To further demonstrate the role of IncHERG in vivo, we performed tumor xenograft assays. We injected $4 \times 10^{6}$ shCtrl or shlncHERG U87 or U251 cells into 6-weekold BALB/c nude mice subcutaneously. At indicative time points, we calculated tumor volumes. We found that lncHERG knockdown greatly delayed tumor propagation in vivo (Figure 4A). Then 7 weeks later, we measured the tumor weights. We found that tumors derived from shlncHERG U87 or U251 cells were lighter and smaller (Figure 4B and 4C). We then collected the formed tumors and checked cell proliferation, migration, invasion and apoptosis by western blot. We found that tumors originated from shlncHERG U87 or U251 cells showed decreased proliferation, migration and invasion but increased
A
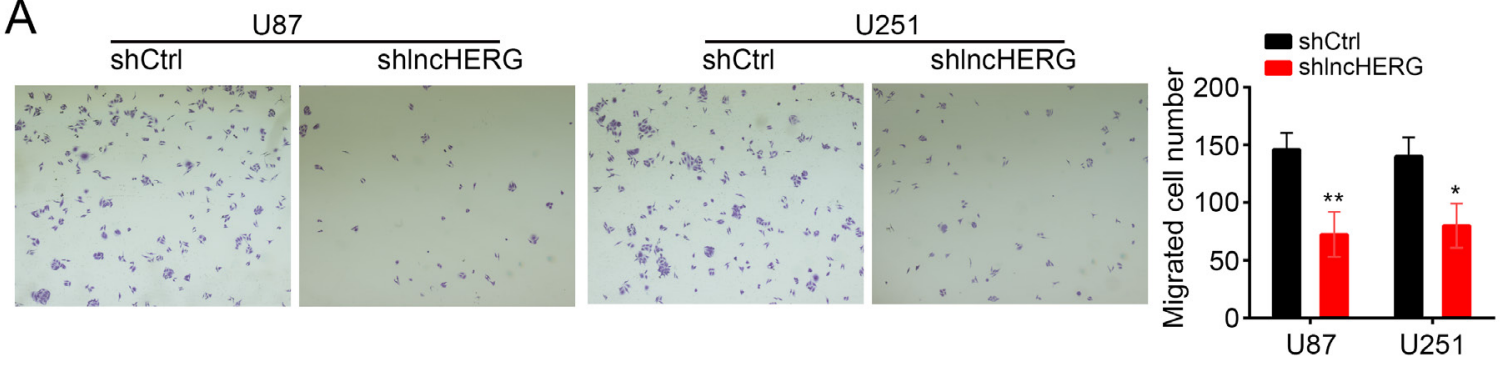

B

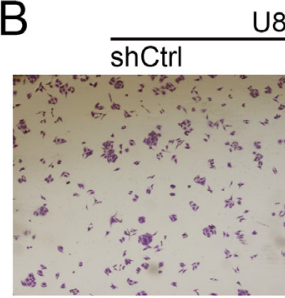
U87 shlncHERG
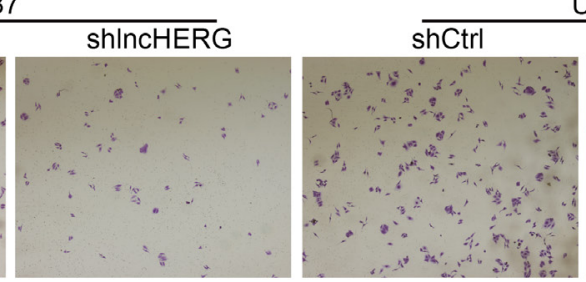

$\mathrm{U} 251$
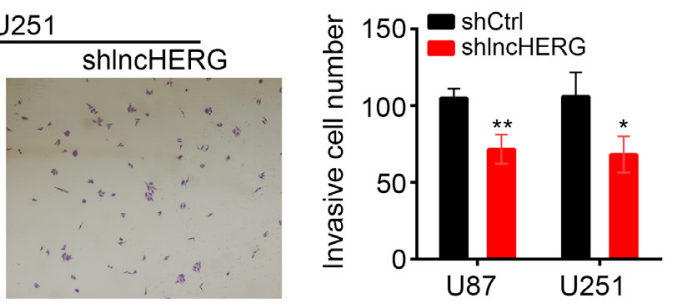

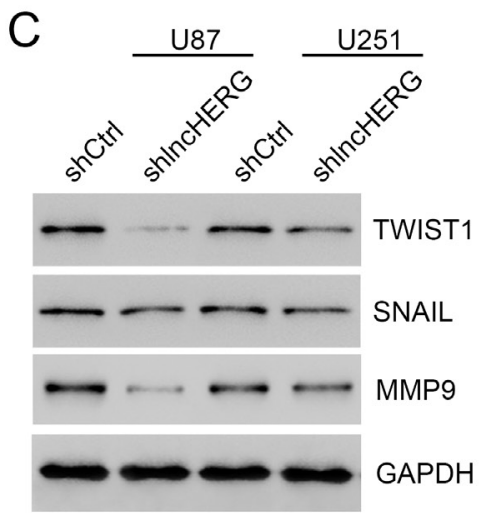

Figure 3: LncHERG enhances cell migration and invasion. (A) LncHERG knockdown seriously inhibited cell migration. (B) LncHERG silence impaired the ability of cell invasion. (C) The expression levels of tumor metastasis-related proteins were measured by WB. LncHERG depletion remarkably inhibited cell metastasis in U87 and U251 cells. All data are representative of three independent experiments and expressed as mean \pm SD. ${ }^{*} p<0.05$ and ${ }^{* *} p<0.01$. 
apoptosis (Figure 4D). Summarily, lncHERG regulated cell proliferation, migration, invasion and apoptosis in vivo.

\section{LncHERG sponges miR-940 in glioblastoma}

Increasing evidences showed that IncRNAs can serve as competitive endogenous RNAs to bind miRNAs [21]. To search the potential binding miRNAs of IncHERG, we made a prediction by a tool (http:// mirdb.org). Some miRNAs were the potential targets of 1ncHERG, but miR-940 scored top. miR-940 was reported to act as a tumor suppressor. For example, miR940 inhibited cell proliferation and apoptosis in ovarian cancer by targeting PKC- $\delta$ [22]. And miR-940 suppresses cell invasion and migration in HCC by targeting CXCR2 [23]. Besides, miR-940 also inhibited triple-negative breast cancer, pancreatic ductal adenocarcinoma and prostate cancer [24-26]. However, the function of miR940 in glioblastoma remains elusive. We found that there are 21 potential binding sites with miR-940 in lncHERG (Figure 5A). We divided lncHERG into 4 truncations (1 500bp, 501 1000bp, 1001 1500bp and 1501 2195bp) and constructed four luciferase reporter plasmid and corresponding mutant plasmids (Figure 1A). We then conducted luciferase activity assays by transfecting pRLlncHERG-wt or pRL-lncHERG-mut together with miR940 mimic or inhibitor into $293 \mathrm{~T}$ cells. We found that miR-940 mimic dramatically inhibited the luciferase activity while miR-940 inhibitor promoted the activity (Figure 5B), which demonstrated that lncHERG can bind to miR-940. Then we transfected miR-940 mimic or inhibitor into U87 or U251 cells and checked mRNA levels of lncHERG. We found that miR-940 mimic inhibited lncHERG expression while miR-940 inhibitor enhanced lncHERG expression (Figure 5C). Consistently, lncHERG knockdown promoted the expression of miR940 while overexpressing $\operatorname{lncHERG}$ inhibited that of miR940 (Figure 5D). Moreover, we found that miR-940 was down-regulated in glioblastoma compared to non-tumor tissues according to an online dataset (GSE90605) (Figure $5 \mathrm{E}$ ). To further confirm it, we analyzed the expression of miR-940 in 80 pairs of glioblastoma tissues and found that miR-940 was also down-regulated in tumor tissues (Figure $5 \mathrm{~F}$ ). Besides, we checked the correlation of lncHERG expression with miR-940 expression in glioblastoma. We found that the expression of IncHERG was negatively correlated with that of miR-940 in 80 glioblastoma samples (Figure 5G). In a word, lncHERG sponged miR940 which was down-regulated in glioblastoma.

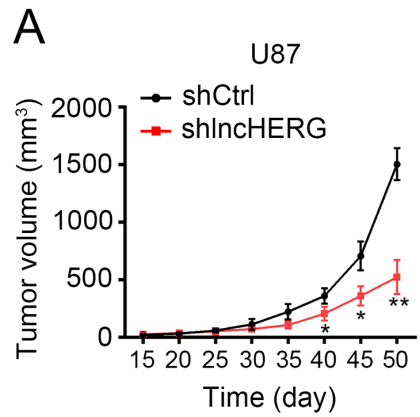

C

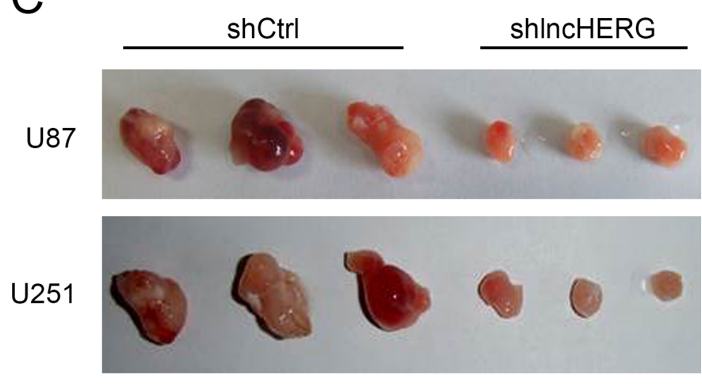

U251

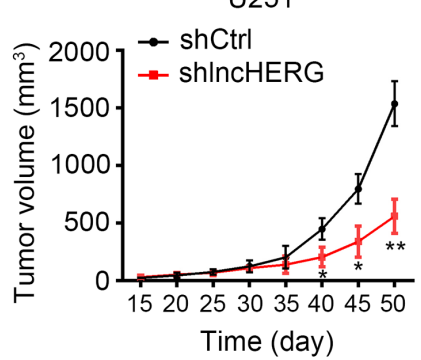

B

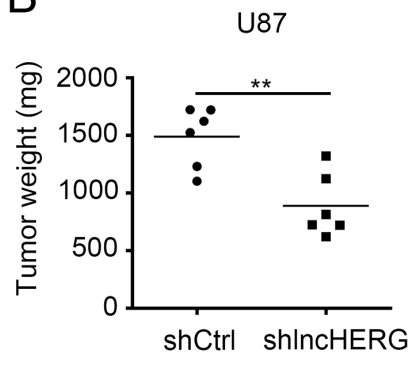

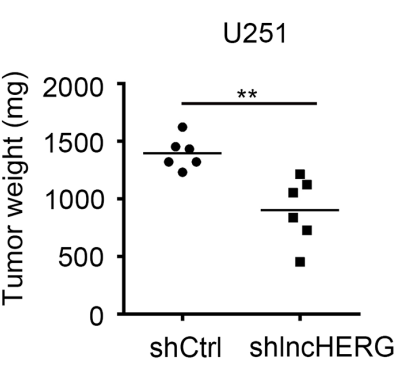

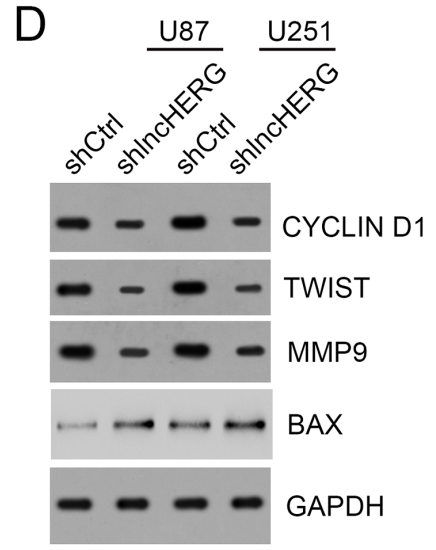

Figure 4: LncHERG depletion impairs tumor propagation in vivo. (A) $4 \times 10^{6}$ shCtrl or sh lncHERG U87 or U251 cells were subcutaneously injected into 6-week-old Balb/c nude mice. The tumor volumes were measured at different time points. IncHERG knockdown remarkably delayed tumor propagation in vivo. (B) and (C) Tumor weights were analyzed 7 weeks post injection. lncHERG knockdown inhibited tumor growth. (D) The proliferation, migration and apoptosis in tumors of C were analyzed by WB. lncHERG depletion seriously inhibited tumor proliferation and migration while promoted apoptosis. All data are representative of three independent experiments and expressed as mean $\pm \mathrm{SD} .{ }^{* *} p<0.01$. 


\section{LncHERG promoted cell proliferation, migration and invasion by sponging miR-940 in glioblastoma}

We have validated that $\operatorname{lncHERG}$ bond to miR-940 in glioblastoma. We wondered whether miR-940 was also a tumor suppressor and whether lncHERG inhibited tumor growth by sponging miR-940 in glioblastoma. To demonstrate it, we conducted MTT assays, we found that lncHERG knockdown inhibited cell proliferation while addition of miR-940 inhibitor reversed it in U87 and 251 cells (Figure 6A). Besides, lncHERG silence impaired colony formation while addition of miR-940 inhibitor in the meantime promoted the ability of colony formation (Figure 6B). Moreover, miR-940 inhibition promoted cell entry into cell cycle even under the condition of IncHERG knockdown (Figure 6C). We also checked the influence of miR-940 on migration and invasion. We found that transfection with miR-940 inhibitor promoted cell migration and invasion while lncHERG knockdown

A

B

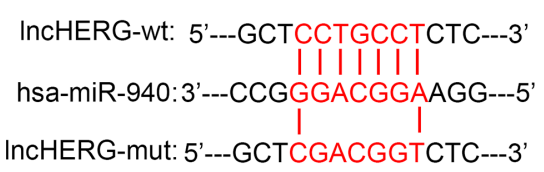

C

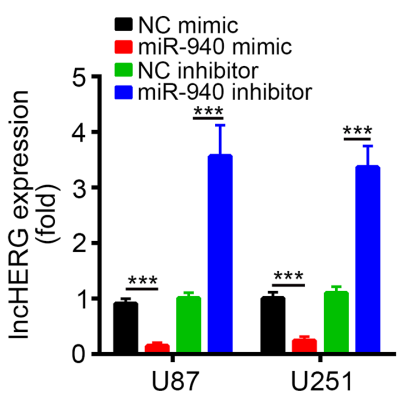

$\mathrm{D}$

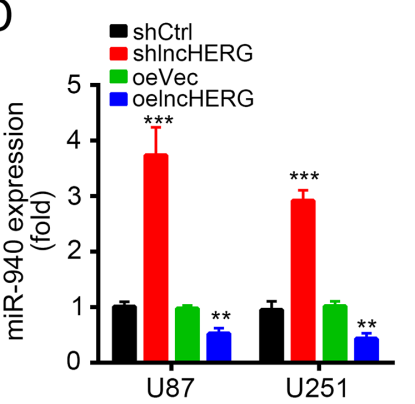

E

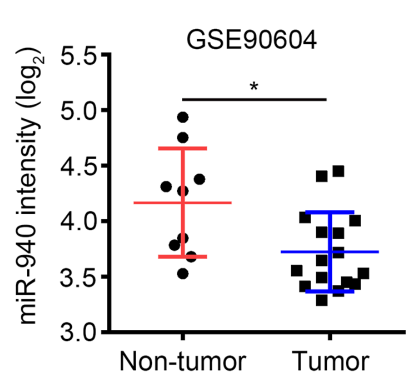

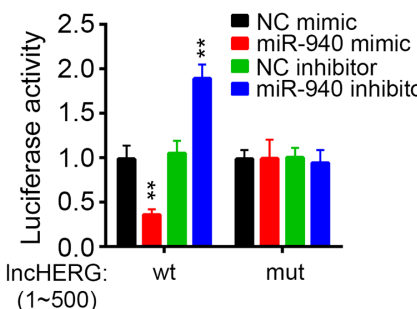
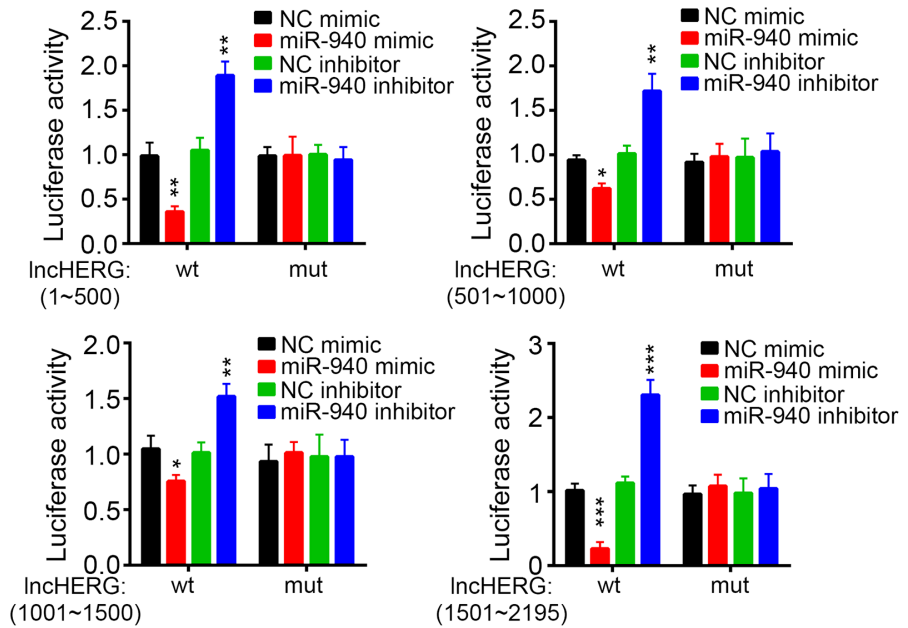

$\mathrm{F}$

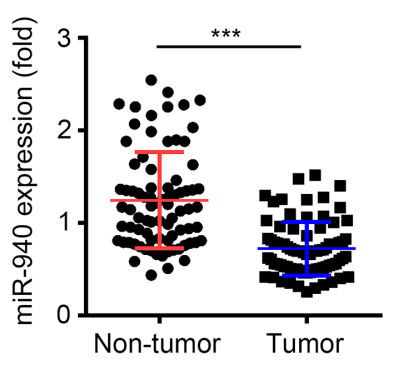

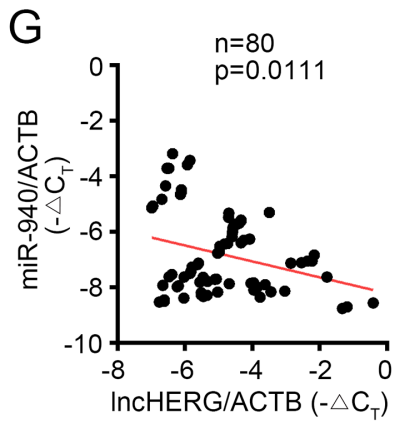

Figure 5: LncHERG sponges miR-940 in glioblastoma. (A) A diagram for the binding site with miR-940 in lncHERG and construction of lncHERG mutant was shown. (B) lncHERG-wt or lncHERG-mut plasmid was transfected into 293T cells with miR-940 mimic or inhibitor for luciferase activity assays. miR-940 mimic remarkably inhibited luciferase activity. (C) miR-940 mimic inhibited the mRNA levels of lncHERG in U87 and U251 cells. (D) lncHERG overexpression inhibited the expression of miR-940 in U87 and U251 cells. (E) miR-940 expression was down-regulated in GBM tissues compared to non-tumor tissues according to a dataset (GSE90604). (F) The expression of miR-940 in 80 GBM samples was analyzed. The expression of miR-940 was down-regulated in GBM tissues compared to peritumor tissues. (G) The expression of lncHERG was negatively correlated with that of miR-940 in GBM tissues. All data are representative of three independent experiments and expressed as mean $\pm \mathrm{SD} .{ }^{*} p<0.05,{ }^{* *} p<0.01$ and ${ }^{* * *} p<0.001$. 
A

U87
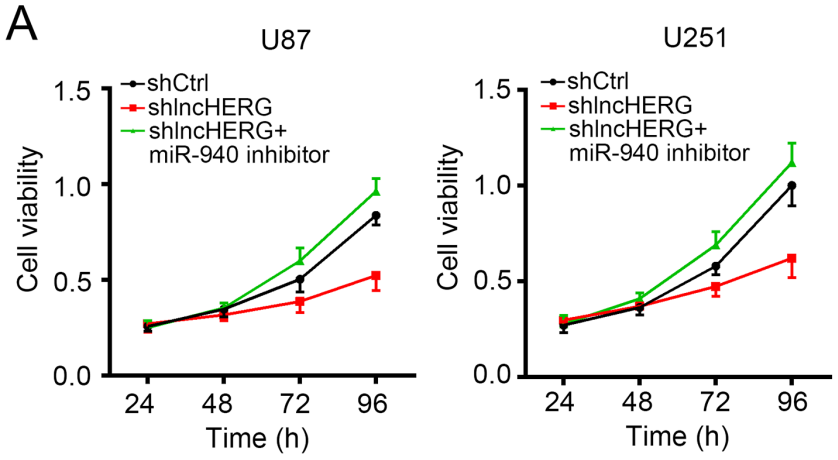

D
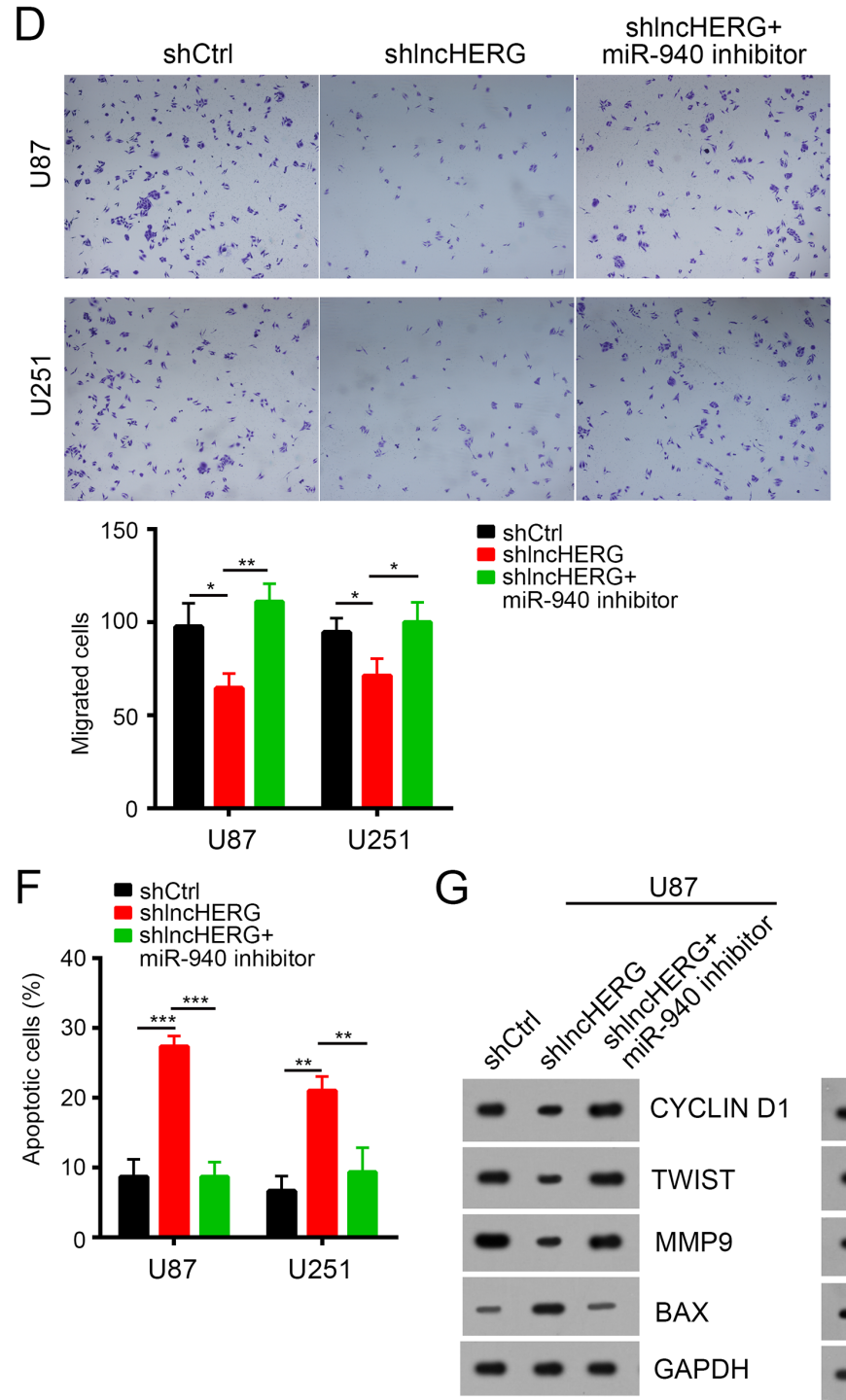
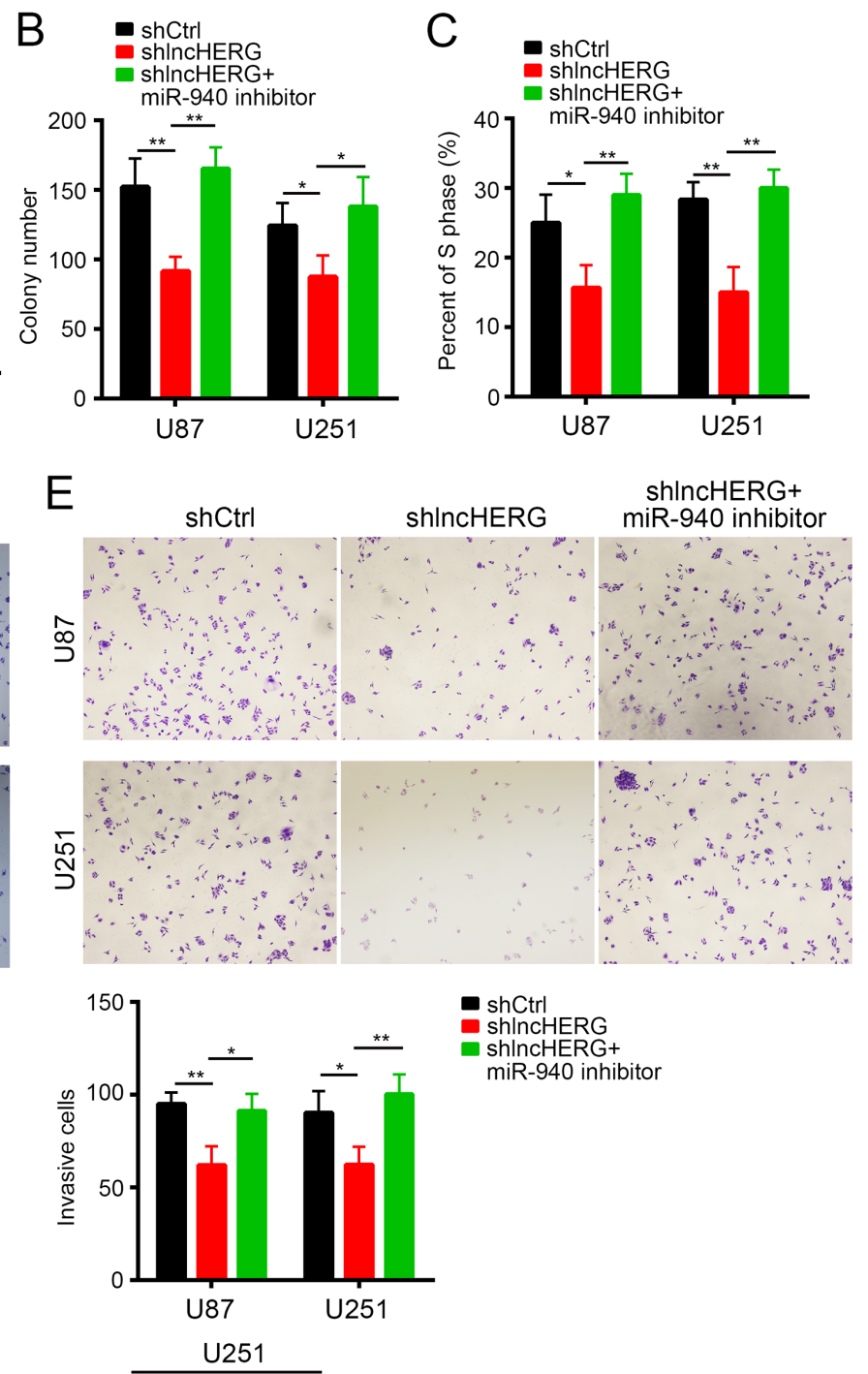

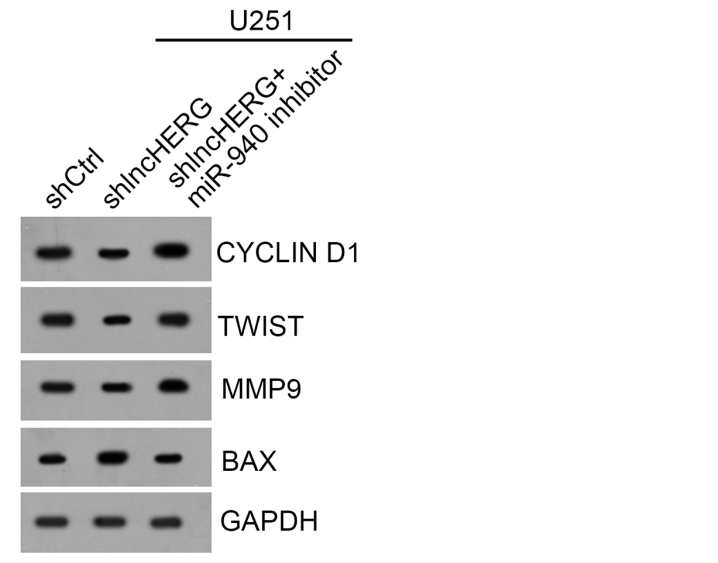

Figure 6: LncHERG promoted cell proliferation, migration and invasion by sponging miR-940 in glioblastoma. (A) lncHERG knockdown inhibited cell proliferation while overexpression of miR-940 mimic in the meantime reversed this trend as shown by MTT assays. (B) lncHERG knockdown inhibited colony formation while overexpression of miR-940 mimic in the meantime promoted colony formation in U87 and U251 cells. (C) lncHERG silence inhibited cell entry into S phase while transfection with miR-940 mimic in the meantime promoted cell division. (D) and (E) lncHERG depletion remarkably inhibited cell migration and invasion while overexpressing miR-940 in the meantime promoted cell migration and invasion. (F) lncHERG knockdown promoted cell apoptosis while miR-940 overexpression in the meantime inhibited cell apoptosis. (G) lncHERG knockdown inhibited cell proliferation, migration and invasion while overexpressing miR-940 in the meantime reversed it in U87 and U251 cells. The protein levels of CYCLIN D1, TWIST, MMP9 and BAX were checked by Western blot. All data are representative of three independent experiments and expressed as mean \pm SD. ${ }^{*} p<0.05,{ }^{* *} p<0.01$ and ${ }^{* * *} p<0.001$. 
inhibited migration and invasion (Figure 6D and 6E). On the other hand, lncHERG depletion promoted cell apoptosis while miR-940 inhibition in the meantime decreased apoptosis (Figure 6F). Taken together, lncHERG promoted cell proliferation, migration and invasion by sponging miR-940 in glioblastoma (Figure 6G).

\section{DISCUSSION}

Glioblastoma was the most common and aggressive brain cancer and gave rise to lots of deaths worldwide every year $[1,2]$. However, the mechanism that regulates glioblastoma development and progression is largely unknown. As the development of bioinformatics, hundreds of IncRNAs have been identified in various tumors [27]. Many lncRNAs are involved in tumorgenesis and can regulate tumor growth and metastasis [16, 28]. In glioblastoma, some lncRNAs have been reported to be essential for tumor development. For example, lncRNA H19 promotes oncogenic effects in glioblastoma [29]. Long non-coding RNA taurine upregulated 1 promotes tumor-induced angiogenesis by inhibiting microRNA-299 in human glioblastoma [30]. IncRNA RP11-838N2.4 promotes the cytotoxic effects of temozolomide in glioblastoma cell lines [31]. However, there are still large amounts of lncRNAs whose function is unknown in glioblastoma. Therefore, in order to develop new and effective therapeutic management of GBM, it is an urgent and necessary to define the molecular mechanism that regulates the genesis of glioblastoma. In our study, we showed an uncharacteristic lncRNA lncHERG that was upregulated in glioblastoma than non-tumor tissues. We showed that lncHERG depletion impaired proliferation, migration and invasion of glioblastoma in vitro and in vivo. What's more, the higher expression of lncHERG in patients with glioblastoma indicated lower survival rate and poorer prognosis. Our study revealed the important role of lncHERG, which may serve as a new prognostic biomarker in glioblastoma.

LncRNAs may exert functions through various mechanisms including regulating chromatin remodeling and binding to miRNAs $[32,33]$. To define the mechanism by which IncHERG exerted function in glioblastoma, we performed a target miRNA prediction. We found that there are 21 potential binding sites with miR-940 in lncHERG, which strongly indicated that lncHERG may sponge miR-940. Moreover, previous studies showed that miR-940 served as a tumor suppressor in various cancers including human nasopharyngeal carcinoma, prostate cancer, pancreatic ductal adenocarcinoma, hepatocellular carcinoma, ovarian cancer and triplenegative breast cancer [22, 24-26, 34, 35]. Nevertheless, the role of miR-940 in glioblastoma is totally unknown. To determine whether miR-940 is also a tumor suppressor in glioblastoma and whether lncHERG promoted tumor growth by inhibiting miR-940, we first conducted luciferase assays. We found that miR-940 mimic inhibited luciferase activity. Besides, we found that miR-940 overexpression decreased the mRNA levels of lncHERG and overexpressing lncHERG also inhibited the expression of miR-940. Moreover, the expression of lncHERG was negatively correlated with that of IncHERG in glioblastoma. All above indicated that lncHERG directly bond to miR-940 in glioblastoma. Then we found that miR-940 was down-regulated in glioblastoma compared to non-tumor tissues, which indicated that miR-940 may also act as a tumor suppressor. To validate it, we performed MTT, colony formation, migration and invasion assays. We found that lncHERG knockdown significant inhibited cell proliferation, migration and invasion, and promoted cell apoptosis. However, addition of miR-940 inhibitor in the meantime fully reversed these trends. Therefore, miR940 acted as a tumor suppressor in glioblastoma and was a target of lncHERG. In different cancers, miR-940 seemed to target respective target. The target genes of miR-940 in glioblastoma remain to be explored further.

In summary, we screened out a unidentified lncRNA lncHERG that regulated tumor development and progression in glioblastoma by directly sponging miR940. Our research revealed the pivot role of $1 \mathrm{ncHERG} /$ miR-940 axis in glioblastoma, which may provide a new sight on the treatment of glioblastoma.

\section{MATERIALS AND METHODS}

\section{Patient samples}

In this study, we obtained 80 pairs of glioblastoma samples from The Second Affiliated Hospital of Zhejiang University School of Medicine. We got consents of approving the usage of these samples in the study from all patients. All the experiments were approved by The Second Affiliated Hospital of Zhejiang University School of Medicine. The study protocol was approved by The Second Affiliated Hospital of Zhejiang University School of Medicine.

\section{Cell lines}

Normal glial cell line (HA) and GBM cell lines (U87, U251 and LN229) were obtained from the Shanghai Cell Bank, Chinese Academy of Sciences (Shanghai, China). HA cells were cultured in Astrocyte Medium (AM). GBM cell lines were cultured in Dulbecco'smodified Eagle medium (DMEM) F12 supplemented with $10 \%$ fetal bovine serum (FBS, Gibco) and $100 \times$ penicillinstreptomycin solution (Invitrogen Life Technologies).

\section{Construction and infection}

Oligonucleotide encoding shRNA targeted lncHERG or a scramble shRNA was obtained from 
Sangon Biotech Inc. (Beijing, China) and constructed into $\mathrm{pLKO}$.1-EGFP vector for lentivirus production. U87 and U251 cells were infected with lentivirus expressing shlncHERG or shCtrl. $\mathrm{GFP}^{+}$stable cell lines were obtained by FACS isolation. shlncHERG sequence was as follows: 5'-GCCTTACAACAGCCTCTTTAC-3'.

\section{Cell proliferation assay}

$2 \times 10^{3}$ cells/well were seeded in a 96-well plate and cultured at $37{ }^{\circ} \mathrm{C}$ for indicated times. Cells proliferation was detected by MTT assays. After cells were cultured for $24 \mathrm{~h}, 48 \mathrm{~h}, 72 \mathrm{~h}$ or $96 \mathrm{~h}$, MTT was added to each group of wells and incubated for $1 \mathrm{~h}$ at $37^{\circ} \mathrm{C}$. Measurement was carried out in absorbance at $490 \mathrm{~nm}$ with a microplate reader according to the manufacturer's instruction.

\section{In vitro migration and invasion assays}

For invasion assay, $1 \times 10^{5}$ cells/well were seeded into the upper well of the Transwell chamber (BD Biosciences) coated with Matrigel (BD Biosciences) and cultured in serum-free DMEM. The lower chamber was filled with DMEM containing $10 \%$ FBS. Cells were incubated at $37^{\circ} \mathrm{C}$ for $48 \mathrm{~h}$ and then fixed with $4 \%$ paraformaldehyde and stained by crystal violet solution for $30 \mathrm{~min}$. Cells on the top surface of the insert were removed with a cotton swab and counted under a microscope in five fields. Similar procedure was also performed for cell migration assay, except that the transwell membranes were not precoated with Matrigel.

\section{Reverse transcription and real-time PCR}

Total RNAs from tumor samples were extracted using Trizol reagent (Life Technologies) according to the Manufacturer's instructions. The cDNA was synthesized from $5 \mu \mathrm{g}$ of RNA using AMV reverse transcriptase (Fermentas, USA). Then real-time quantitative PCR was conducted using a standard SYBR Green PCR kit (Thermo Fisher Scientific, Rockford, IL, USA) on an ABI 7300 Real-Time PCR machine (Applied Biosystems, Foster City, CA, USA). The primer sequences were as follows: lncHERG forward: 5'-TTTTTGTGCCTGCCTCGTTG-3'; lncHERG reverse: 5'-GTTCGGCCTCAAGAAACTGC-3'; miR-940 forward: 5'-CCTGTCTTACTTTTCCGAAGGAC-3'; miR-940 reverse: 5'-TTGCTGTATTGTTGCCCATGT-3'.

\section{In vivo assay}

6-week-old BALB/c nude mice were from HFK Biosciences. $4 \times 10^{6}$ shCtrl or shlncHERG tumor cells were subcutaneously injected into nude mice. Tumor volumes were analyzed at indicative time points. And the tumor weights were measured 7 weeks after injection. Animal experiments were performed in accordance with relevant guidelines and regulations of the Institutional Animal Care and Use Committees at The Second Affiliated Hospital of Zhejiang University School of Medicine, and protocols were approved by the Institutional Animal Care and Use Committees at The Second Affiliated Hospital of Zhejiang University School of Medicine.

\section{Luciferase reporter assay}

$293 \mathrm{~T}$ cells were seeded into a $24-w e l l$ plate. For lncHERG and miR-940 interaction, cells were cotransfected with wild-type, mutated IncHERG reporter plasmid or pRL-TK vector, and miR-940 mimics or miR940 inhibitor. Luciferase assays were conducted $24 \mathrm{~h}$ after transfection using the Dual Luciferase Reporter Assay System (Promega, WI, USA).

\section{In situ hybridization}

LncHERG expression in GBM tissues were analyzed using biotin-labeled specific lncHERG probes. Paraffinized sections were deparaffinized with xylene and $100 \%$ ethanol. Then sections were incubated with biotinlabeled probes for $18 \mathrm{~h}$ at $40{ }^{\circ} \mathrm{C}$. DAB substrate was used for colorimetric detection of lncHERG. Finally, the sections were co-stained with hematoxylin, followed by dehydration in graded alcohols and xylene. IncHERG probe sequences as follows: \#1: 5'-GGCAGGCACAAAAATGGTCT-3'; \#2: 5'-AGGAACGTGGTCTGGAAGGC-3'.

\section{Northern blot}

RNAs were extracted from GBM samples using TRIZOL (Invitrogen). LncHERG and GAPHD probes for Northern blot were achieved by Biotin RNA labeling mix (Roche). The RNA samples were separated by electrophoresis and transferred to NC membrane. Then the membranes were incubated with hydration buffer containing probes. Finally, RNA signal was detected with Chemiluminescent Nucleic Acid Detection Module (Thermo Scientific).

\section{Statistical analysis}

All statistical analyses were performed using SPSS 20.0 (IBM, SPSS, Chicago, IL, USA) and GraphPad Prism. Student's t-test and one-way ANOVA were used to analyze 2 or multiple groups, respectively, for statistical significance. Pearson correlation coefficient analysis was used to determine the correlations. The overall survival curves were calculated with the Kaplan-Meier method and were analyzed with the log-rank test. $\mathrm{P}<0.05$ was considered statistically significant in all cases.

\section{Author contributions}

J.S. designed and performed experiments, analyzed data and wrote the paper; Y.W., C.S., B.Q. and Y.Z. 
performed experiments; G.C. and J.S. initiated the study, organized, designed, and wrote the paper.

\section{CONFLICTS OF INTEREST}

The authors declare no competing financial interests.

\section{FUNDING}

This work was supported by the Natural Science Foundation of Zhejiang Province (LY16H090005).

\section{REFERENCES}

1. Stupp R, Mason WP, van den Bent MJ, Weller M, Fisher B, Taphoorn MJ, Belanger K, Brandes AA, Marosi C, Bogdahn U, Curschmann J, Janzer RC, Ludwin SK, et al. Radiotherapy plus concomitant and adjuvant temozolomide for glioblastoma. N Engl J Med. 2005; 352:987-96.

2. Lee JH, Jung TY, Jung S, Kim IY, Jang WY, Moon KS, Jeong EH. Performance status during and after radiotherapy plus concomitant and adjuvant temozolomide in elderly patients with glioblastoma multiforme. J Clin Neurosci. 2013; 20:503-8.

3. Kuhnt D, Becker A, Ganslandt O, Bauer M, Buchfelder M, Nimsky C. Correlation of the extent of tumor volume resection and patient survival in surgery of glioblastoma multiforme with high-field intraoperative MRI guidance. Neuro Oncol. 2011; 13:1339-48.

4. Oppenlander ME, Wolf AB, Snyder LA, Bina R, Wilson JR, Coons SW, Ashby LS, Brachman D, Nakaji P, Porter RW, Smith KA, Spetzler RF, Sanai N. An extent of resection threshold for recurrent glioblastoma and its risk for neurological morbidity. J Neurosurg. 2014; 120:846-53.

5. Liang BC, Thornton AF Jr, Sandler HM, Greenberg HS. Malignant astrocytomas: focal tumor recurrence after focal external beam radiation therapy. J Neurosurg. 1991; 75:559-63.

6. Wu F, Zhang C, Cai J, Yang F, Liang T, Yan X, Wang H, Wang W, Chen J, Jiang T. Upregulation of long noncoding RNA HOXA-AS3 promotes tumor progression and predicts poor prognosis in glioma. Oncotarget. 2017; 8:53110-23. https://doi.org/10.18632/oncotarget.18162.

7. Zhang K, Sun X, Zhou X, Han L, Chen L, Shi Z, Zhang A, Ye M, Wang Q, Liu C, Wei J, Ren Y, Yang J, et al. Long non-coding RNA HOTAIR promotes glioblastoma cell cycle progression in an EZH2 dependent manner. Oncotarget. 2015; 6:537-46. https://doi.org/10.18632/ oncotarget.2681.

8. Sadetzki S, Zach L, Chetrit A, Nass D, Hoffmann C, Ram Z, Zaaroor M, Umansky F, Rappaport ZH, Cohen A, Wald U, Rothman S, Hadani M. Epidemiology of gliomas in Israel: a nationwide study. Neuroepidemiology. 2008; 31:264-9.

9. Ulitsky I, Bartel DP. lincRNAs: genomics, evolution, and mechanisms. Cell. 2013; 154:26-46.
10. Li J, Han W, Shen X, Han S, Ye H, Huang G. DNA methylation signature of long noncoding RNA genes during human pre-implantation embryonic development. Oncotarget. 2017; 8:56829-38. https://doi.org/10.18632/ oncotarget.18072.

11. Liu B, Ye B, Yang L, Zhu X, Huang G, Zhu P, Du Y, Wu J, Qin X, Chen R, Tian Y, Fan Z. Long noncoding RNA $\operatorname{lncKdm} 2 \mathrm{~b}$ is required for ILC3 maintenance by initiation of Zfp292 expression. Nat Immunol. 2017; 18:499-508.

12. Li W, Li N, Kang X, Shi K, Chen Q. Prognostic value of the long noncoding RNA HOTTIP in human cancers. Oncotarget. 2017; 8:59563-9. https://doi.org/10.18632/ oncotarget.19166.

13. Yang Y, Qian J, Xiang Y, Chen Y, Qu J. The prognostic value of long noncoding RNA HOTTIP on clinical outcomes in breast cancer. Oncotarget. 2017; 8:6833-44. https://doi.org/10.18632/oncotarget.14304.

14. Batista PJ, Chang HY. Long noncoding RNAs: cellular address codes in development and disease. Cell. 2013; 152:1298-307.

15. Ponting CP, Oliver PL, Reik W. Evolution and functions of long noncoding RNAs. Cell. 2009; 136:629-41.

16. Shi X, Zhang H, Wang M, Xu X, Zhao Y, He R, Zhang M, Zhou M, Li X, Peng F, Shi C, Shen M, Wang X, et al. LncRNA AFAP1-AS1 promotes growth and metastasis of cholangiocarcinoma cells. Oncotarget. 2017; 8:58394-404. https://doi.org/10.18632/oncotarget.16880.

17. Ma HW, Xie M, Sun M, Chen TY, Jin RR, Ma TS, Chen QN, Zhang EB, He XZ, De W, Zhang ZH. The pseudogene derived long noncoding RNA DUXAP8 promotes gastric cancer cell proliferation and migration via epigenetically silencing PLEKHO1 expression. Oncotarget. 2017; 8:52211-24. https://doi.org/10.18632/oncotarget.11075.

18. Cai Q, Jin L, Wang S, Zhou D, Wang J, Tang Z, Quan Z. Long non-coding RNA UCA1 promotes gallbladder cancer progression by epigenetically repressing p21 and E-cadherin expression. Oncotarget. 2017; 8:47957-68. https://doi.org/10.18632/oncotarget.18204.

19. Zhan Y, Li Y, Guan B, Chen X, Chen Z, He A, He S, Gong Y, Peng D, Liu Y, Cai Z, Li X, Zhou L. Increased expression of long non-coding RNA CCEPR is associated with poor prognosis and promotes tumorigenesis in urothelial bladder carcinoma. Oncotarget. 2017; 8:44326-34. https://doi. org/10.18632/oncotarget.17872.

20. Brodie S, Lee HK, Jiang W, Cazacu S, Xiang C, Poisson LM, Datta I, Kalkanis S, Ginsberg D, Brodie C. The novel long non-coding RNA TALNEC2, regulates tumor cell growth and the stemness and radiation response of glioma stem cells. Oncotarget. 2017; 8:31785-801. https://doi. org/10.18632/oncotarget.15991.

21. Tian Z, Wen S, Zhang Y, Shi X, Zhu Y, Xu Y, Lv H, Wang G. Identification of dysregulated long non-coding RNAs/ microRNAs/mRNAs in TNM I stage lung adenocarcinoma. Oncotarget. 2017; 8:51703-18. https://doi.org/10.18632/ oncotarget.18512. 
22. Wang F, Wang Z, Gu X, Cui J. miR-940 upregulation suppresses cell proliferation and induces apoptosis by targeting PKC-delta in ovarian cancer OVCAR3 cells. Oncol Res. 2017; 25:107-14.

23. Ding D, Zhang Y, Yang R, Wang X, Ji G, Huo L, Shao Z, Li $\mathrm{X}$. miR-940 suppresses tumor cell invasion and migration via regulation of CXCR2 in hepatocellular carcinoma. Biomed Res Int. 2016; 2016:7618342.

24. Hou L, Chen M, Yang H, Xing T, Li J, Li G, Zhang L, Deng $\mathrm{S}, \mathrm{Hu}$ J, Zhao X, Jiang J. MiR-940 inhibited cell growth and migration in triple-negative breast cancer. Med Sci Monit. 2016; 22:3666-72.

25. Song B, Zhang C, Li G, Jin G, Liu C. MiR-940 inhibited pancreatic ductal adenocarcinoma growth by targeting MyD88. Cell Physiol Biochem. 2015; 35:1167-77.

26. Rajendiran S, Parwani AV, Hare RJ, Dasgupta S, Roby RK, Vishwanatha JK. MicroRNA-940 suppresses prostate cancer migration and invasion by regulating MIEN1. Mol Cancer. 2014; 13:250.

27. Prensner JR, Chinnaiyan AM. The emergence of lncRNAs in cancer biology. Cancer Discov. 2011; 1: 391-407.

28. Tao Y, Han T, Zhang T, Ma C, Sun C. LncRNA CHRFinduced miR-489 loss promotes metastasis of colorectal cancer via TWIST1/EMT signaling pathway. Oncotarget. 2017; 8:36410-22. https://doi.org/18632/oncotarget.16850.

29. Wu W, Hu Q, Nie E, Yu T, Wu Y, Zhi T, Jiang K, Shen F, Wang Y, Zhang J, You Y. Hypoxia induces H19 expression through direct and indirect Hif-1alpha activity, promoting oncogenic effects in glioblastoma. Sci Rep. 2017; 7:45029.
30. Cai H, Liu X, Zheng J, Xue Y, Ma J, Li Z, Xi Z, Li Z, Bao M, Liu Y. Long non-coding RNA taurine upregulated 1 enhances tumor-induced angiogenesis through inhibiting microRNA-299 in human glioblastoma. Oncogene. 2017; 36:318-31.

31. Liu Y, Xu N, Liu B, Huang Y, Zeng H, Yang Z, He Z, Guo H. Long noncoding RNA RP11-838N2.4 enhances the cytotoxic effects of temozolomide by inhibiting the functions of miR-10a in glioblastoma cell lines. Oncotarget. 2016; 7:43835-51. https://doi.org/10.18632/ oncotarget.9699.

32. Zampetaki A, Mayr M. Long noncoding RNAs and angiogenesis: regulatory information for chromatin remodeling. Circulation. 2017; 136:80-2.

33. Tran DD, Kessler C, Niehus SE, Mahnkopf M, Koch A, Tamura T. Myc target gene, long intergenic noncoding RNA, Linc00176 in hepatocellular carcinoma regulates cell cycle and cell survival by titrating tumor suppressor microRNAs. Oncogene. 2017.

34. Ma J, Sun F, Li C, Zhang Y, Xiao W, Li Z, Pan Q, Zeng H, Xiao G, Yao K, Hong A, An J. Depletion of intermediate filament protein Nestin, a target of microRNA-940, suppresses tumorigenesis by inducing spontaneous DNA damage accumulation in human nasopharyngeal carcinoma. Cell Death Dis. 2014; 5:e1377.

35. Yuan B, Liang Y, Wang D, Luo F. MiR-940 inhibits hepatocellular carcinoma growth and correlates with prognosis of hepatocellular carcinoma patients. Cancer Sci. 2015; 106:819-24. 